\title{
Criminologie
}

\section{Réflexions sur la réalité actuelle des détenues dans le système correctionnel fédéral}

\section{Marie-Andrée Cyrenne}

Volume 35, numéro 2, automne 2002

Femmes et enfermement au Canada : une décennie de réformes

URI : https://id.erudit.org/iderudit/008289ar

DOI : https://doi.org/10.7202/008289ar

Aller au sommaire du numéro

\section{Éditeur(s)}

Les Presses de l'Université de Montréal

ISSN

0316-0041 (imprimé)

1492-1367 (numérique)

Découvrir la revue

Citer cet article

Cyrenne, M.-A. (2002). Réflexions sur la réalité actuelle des détenues dans le système correctionnel fédéral. Criminologie, 35(2), 31-52.

https://doi.org/10.7202/008289ar
Résumé de l'article

Suite à l'acceptation par le gouvernement du rapport La création de choix, le Service correctionnel du Canada (SCC) a ouvert quatre établissements régionaux pour les femmes ainsi qu'un pavillon de ressourcement pour les détenues autochtones. Ces établissements ont été conçus en fonction d'un modèle opérationnel basé sur la prise en charge par les délinquantes de leur incarcération et de leur vie. Des programmes répondant aux besoins uniques des délinquantes ont été élaborés et mis en place. D’ailleurs, un des aspects novateurs s'avère la possibilité, pour certaines détenues, de vivre avec leur(s) enfant(s) à l'intérieur du pénitencier. De plus, le SCC met en oeuvre présentement une stratégie d'intervention intensive pour les détenues présentant des problèmes de santé mentale et pour celles qui ont un classement sécuritaire maximum. En communauté, la grande majorité des délinquantes complètent avec succès leur période de surveillance ; le SCC s'est de plus doté, au cours des dernières années, d'un réseau de logement mieux adapté à leurs besoins. Le moment est donc propice à une réflexion sur la réalité actuelle des délinquantes dans le système fédéral.
Ce document est protégé par la loi sur le droit d'auteur. L’utilisation des services d’Érudit (y compris la reproduction) est assujettie à sa politique d'utilisation que vous pouvez consulter en ligne.

https://apropos.erudit.org/fr/usagers/politique-dutilisation/ 


\title{
Réflexions sur la réalité actuelle des détenues dans le système correctionnel fédéral
}

\author{
Marie-Andrée Cyrenne \\ Directrice générale intérimaire \\ Secteur des délinquantes \\ Service correctionnel du Canada. \\ cyrennema@csc-scc.gc.ca
}

En collaboration avec le personnel du Secteur des délinquantes

Administration centrale du Service correctionnel du Canada

RÉSUMÉ • Suite à l'acceptation par le gouvernement du rapport La création de choix, le Service correctionnel du Canada (SCC) a ouvert quatre établissements régionaux pour les femmes ainsi qu'un pavillon de ressourcement pour les détenues autochtones. Ces établissements ont été conçus en fonction d'un modèle opérationnel basé sur la prise en charge par les délinquantes de leur incarcération et de leur vie. Des programmes répondant aux besoins uniques des délinquantes ont été élaborés et mis en place. D'ailleurs, un des aspects novateurs s'avère la possibilité, pour certaines détenues, de vivre avec leur(s) enfant(s) à l'intérieur du pénitencier. De plus, le SCC met en œuvre présentement une stratégie d'intervention intensive pour les détenues présentant des problèmes de santé mentale et pour celles qui ont un classement sécuritaire maximum. En communauté, la grande majorité des délinquantes complètent avec succès leur période de surveillance; le SCC s'est de plus doté, au cours des dernières années, d'un réseau de logement mieux adapté à leurs besoins. Le moment est donc propice à une réflexion sur la réalité actuelle des délinquantes dans le système fédéral.

\begin{abstract}
Following the acceptation by the government of the report Creating Choices, the Correctional Service of Canada (CSC) opened four regional institutions for women and a healing lodge for Aboriginal women inmates. The design of these institutions was based on an operational model that gives offenders control over their incarceration and their lives. Programs that meet the unique needs of women were developed and implemented. One innovation is giving some inmates the possibility of living with their child(ren) within the institution. Furthermore, CSC is currently implementing the Intensive Intervention Strategy for inmates with mental health problems and for those classified as maximum-security. In the community, the vast majority of offenders successfully complete their period of supervision and, in recent years, CSC has developed a housing network better suited to their needs. Thus, this is now an opportune moment to reflect on the current realities of women offenders in the federal system.
\end{abstract}




\section{Introduction}

Des changements significatifs au niveau correctionnel fédéral sont survenus durant la dernière décennie. Certains documents, dont la Loi sur le système correctionnel et la mise en liberté sous condition (LSCMLC, 1992), La Mission du Service correctionnel du Canada (SCC, 1997), le Rapport La création de choix (Rapport du groupe d'étude sur les femmes purgeant une peine fédérale, 1990) ainsi que le Rapport Arbour, Commission d'enquête sur certains événements survenus à la Prison des femmes de Kingston (L'honorable Louise Arbour, 1996) pour n'en nommer que quelques-uns, constituent des éléments-clés de ce cheminement. Ils doivent être pris en considération pour une meilleure compréhension de la façon dont le SCC gère le problème des délinquantes.

La $L S C M L C$ encadre les pratiques du SCC et dicte clairement ses obligations au chapitre du respect des genres, des ethnies et des différences culturelles et linguistiques. De façon bien spécifique, elle enjoint au Service de consulter régulièrement les groupes d'intérêt aussi bien que les experts ayant une expérience de travail auprès des délinquantes pour l'élaboration de programmes qui sont destinés à ces dernières.

Dans son énoncé de mission, le SCC s'engage à répondre aux besoins particuliers des délinquantes et des autochtones. De plus, le SCC reconnaît «que la collectivité est le meilleur environnement dans lequel un délinquant peut démontrer pleinement sa capacité de fonctionner en tant que citoyen respectueux des lois» (p. 10). À cet égard, le SCC fournira «les programmes, l'aide et la surveillance qu'il faut pour permettre leur libération graduelle, et ce, aussitôt qu'une libération peut être effectuée sans porter atteinte à la sécurité» (p. 10).

Un point marquant en matière correctionnelle pour les délinquantes fut l'acceptation en 1990, par le gouvernement, du Rapport La création de choix. Ce rapport recommandait certains changements importants, soit la fermeture de la prison des femmes située à Kingston en Ontario (seul pénitencier pour les détenues purgeant une sentence de deux ans et plus), l'ouverture d'établissements régionaux et d'un pavillon de ressourcement, ainsi que le développement d'une stratégie de mise en liberté pour les délinquantes.

Durant la dernière décennie, le SCC a décentralisé la population des délinquantes par l'ouverture d'établissements régionaux, a mis en œuvre un pavillon de ressourcement pour les détenues autochtones, a établi un régime correctionnel axé sur la vie en unité d'habitation, a mis sur pied des programmes répondant aux besoins uniques des délinquantes, et a 
élaboré une stratégie d'intervention intensive pour les détenues présentant des problèmes de santé mentale et pour celles qui ont un classement sécuritaire maximum. Quant à la prison des femmes, elle a fermé officiellement ses portes le 6 juillet 2000 .

Après plus de dix ans d'évolution en matière correctionnelle, le moment est propice à une réflexion sur la réalité actuelle des délinquantes dans le système fédéral. Cet article tentera d'établir un profil de la population carcérale féminine purgeant une sentence fédérale, de décrire le fonctionnement des établissements régionaux et du pavillon de ressourcement, d'aborder le sujet des délinquantes en libération conditionnelle et de cerner les enjeux et les défis des prochaines années. Dans le cadre de cet article, les données utilisées ou les documents cités ont été majoritairement produits par le personnel du SCC ou par des personnesressources engagées par le SCC.

\section{Qui sont les délinquantes?}

Le tableau 1 illustre l'évolution de la population carcérale fédérale, soit les détenues purgeant une peine de deux ans et plus, entre septembre 1996 et septembre 2001 et ce, pour chacune des régions. Au niveau administratif, le SCC se divise en cinq régions, comme l'indique le tableau. La région de l'Atlantique se compose des quatre provinces maritimes, la région de l'Ontario comprend la province de l'Ontario et le Nunavut, la région des Prairies regroupe les provinces du Manitoba, de la Saskatchewan, de l'Alberta et les Territoires du Nord-Ouest, et la région du Pacifique comprend la Colombie-Britannique et le Yukon.

TA B LEA U 1

Tendances - Population incarcérée

\begin{tabular}{|l|c|c|c|c|c|c|}
\hline & Atlantique & Québec & Ontario & Prairies & Pacifique & Total \\
\hline Sept. 1996 & 26 & 54 & 134 & 82 & 33 & 329 \\
\hline Sept. 1997 & 35 & 66 & 99 & 81 & 33 & 314 \\
\hline Sept. 1998 & 32 & 63 & 119 & 83 & 32 & 329 \\
\hline Sept. 1999 & 35 & 64 & 103 & 120 & 32 & 354 \\
\hline Sept. 2000 & 51 & 60 & 94 & 120 & 33 & 358 \\
\hline Sept. 2001 & 42 & 63 & 93 & 138 & 34 & 370 \\
\hline
\end{tabular}


Sur le plan national, le nombre de femmes incarcérées est passé de 329 à 370 entre septembre 1996 et septembre 2001, avec des augmentations plus significatives dans les régions de l'Atlantique et des Prairies.

Cependant, comparativement à la population masculine, les détenues sont peu nombreuses. Elles représentent environ 3\% de l'ensemble de la population carcérale fédérale. Selon nos données, le profil des délinquantes incarcérées à l'automne 2001 se résumait comme suit :

- plus de la moitié des délinquantes sont âgées de 18 à 34 ans;

- les délinquantes autochtones représentent environ le quart de la population carcérale féminine;

- en ce qui a trait aux délits, plus de $65 \%$ des délinquantes purgent une sentence pour un crime violent, soit $18 \%$ pour meurtre au premier et au deuxième degré et $49 \%$ pour un autre crime ayant une composante violente dont, par ordre d'importance, le vol à main armée, l'homicide involontaire et les voies de faits graves;

- $35 \%$ des délinquantes ont commis un crime n'ayant pas de composante directe de violence dont, par ordre d'importance, le trafic de stupéfiants, l'importation/exportation de stupéfiants et la possession en vue d'un trafic;

- environ le tiers des détenues séjournent en établissement pour une sentence de moins de trois ans, alors qu'environ $19 \%$ d'entre elles y sont pour une sentence à perpétuité ou indéterminée ;

- environ $80 \%$ des délinquantes purgent leur première sentence fédérale.

Les chiffres présentés doivent être interprétés comme des indicateurs. La population carcérale féminine étant si peu nombreuse, il faut analyser les données sur plusieurs années afin de cerner les tendances et ainsi tracer un portrait plus juste de la clientèle. Mais déjà ces chiffres démontrent la grande diversité des besoins auxquels les programmes et services des établissements régionaux doivent répondre.

\section{Les établissements régionaux}

Les établissements régionaux sont les suivants : établissement Nova pour femmes situé à Truro (Nouvelle-Écosse); établissement de Joliette situé à Joliette (Québec); établissement Grand Valley pour femmes situé à Kitchener (Ontario); établissement d'Edmonton pour femmes situé à Edmonton (Alberta). En Colombie-Britannique, le Centre correctionnel 
pour femmes de Burnaby, prison provinciale, héberge, en vertu d'une entente fédérale-provinciale, les détenues purgeant une sentence de deux ans et plus ${ }^{1}$.

Les établissements régionaux ont été conçus en fonction d'un modèle opérationnel basé sur la prise en charge par les délinquantes de leur incarcération et de leur vie. Les détenues sont logées dans des unités d'habitation autonomes regroupées derrière le bâtiment principal. Chaque unité peut accueillir jusqu'à dix femmes et comprend cuisine, salle à manger, salle commune et chambres individuelles ou à occupation double. Les résidentes ont libre accès à la cour intérieure durant la journée et en début de soirée.

Elles sont responsables de l'entretien ménager, de leur buanderie ainsi que de la préparation des repas. Selon le nombre de résidentes, chaque unité d'habitation se voit accorder un budget hebdomadaire pour l'épicerie. En fonction d'une liste de prix, elles doivent planifier leur menu et déterminer les articles à commander tout en respectant le budget alloué. Cette façon de procéder leur permet d'influencer la gestion des repas et de pouvoir planifier des menus plus élaborés pour célébrer certaines occasions spéciales. La vie en unité d'habitation a pour objectif général de maintenir un niveau de responsabilité similaire à ce qui se passe à l'extérieur du pénitencier.

Le bâtiment principal regroupe, outre les locaux administratifs, les locaux réservés à l'éducation, aux programmes, aux loisirs, aux visites et aux services de santé. Les mesures de sécurité comprennent principalement une clôture de huit pieds avec fils barbelés, un système de détection permettant de signaler les évasions et les intrusions, ainsi qu'un système de caméras.

Cependant, en matière de sécurité, l'accent est mis sur la sécurité dynamique. Par sécurité dynamique, on entend toute activité qui contribue à promouvoir un environnement sécuritaire par le maintien de relations interpersonnelles constructives. Ce concept repose sur la prémisse que les relations positives entre le personnel et les détenues font partie intégrante de l'élaboration d'un régime correctionnel axé sur le respect des droits et de la dignité des détenues, tout en soutenant un milieu exempt de violence.

1. En janvier 2002, le gouvernement provincial de la Colombie-Britannique a annoncé la fermeture de huit prisons incluant le Centre correctionnel pour femmes de Burnaby. Cette fermeture est prévue pour mars 2004. Le SCC étudie actuellement différentes options afin de continuer l'hébergement des délinquantes fédérales à l'intérieur de leur province. 
De plus, les intervenants de première ligne intègrent les deux aspects du travail correctionnel, soit la relation d'aide et le contrôle, en ce sens qu'ils ont des tâches de gestion de cas tout en devant accomplir des fonctions traditionnellement liées à la sécurité, telles que les fouilles et les dénombrements. En effet, ils sont responsables de la rédaction et du suivi du plan correctionnel, incluant la production des rapports nécessaires à la présentation des cas aux audiences de la Commission nationale des libérations conditionnelles.

La sélection et la formation du personnel s'avèrent donc des éléments essentiels pour le développement et le maintien du modèle correctionnel préconisé dans les établissements régionaux. C'est pourquoi le processus de sélection des intervenants de première ligne est distinct de celui qui s'applique aux établissements pour hommes; distinct, en ce sens que s'ajoutent aux connaissances requises pour travailler dans un établissement correctionnel, l'évaluation des connaissances reliées à la délinquance féminine et des aptitudes à travailler avec cette clientèle.

De plus, une formation d'une durée de dix jours, axée sur la délinquance féminine, a été élaborée et fait partie de la formation obligatoire que doit recevoir le personnel travaillant dans les établissements pour femmes. Cette formation touche, notamment, la criminalité chez les femmes, l'automutilation et le suicide, les problèmes de santé mentale, la gestion des conflits et la médiation, ainsi que plusieurs autres sujets d'intérêt.

\section{Pavillon de ressourcement}

Alors que les autochtones représentent moins de $3 \%$ de la population canadienne, ils constituent environ $15 \%$ des délinquants et des délinquantes sous responsabilité fédérale. Pour ce qui est des délinquantes autochtones, leur surreprésentation est croissante depuis 1995, étant passée graduellement de $16 \%$ à $25 \%$ de la population carcérale féminine (automne 2001). À cette date, 67 des 89 délinquantes autochtones incarcérées venaient de la région des Prairies.

Le pavillon de ressourcement Okimaw Ohci, situé à Maple Creek (Saskatchewan), a été conçu en collaboration avec des représentants des communautés autochtones afin d'offrir un milieu de vie unique aux délinquantes voulant s'investir au niveau d'un processus de guérison. Le pavillon de ressourcement est guidé par les enseignements et les traditions autochtones. 
La structure de l'établissement et le cadre naturel s'unissent pour offrir aux résidentes un environnement propice au ressourcement. Le bâtiment réservé aux pratiques spirituelles, construit selon le modèle de tipi, est le lieu de rassemblement privilégié où les échanges et le partage font partie intégrante du processus de guérison. La présence d'aînés en tout temps sur le site constitue un des éléments centraux du pavillon de ressourcement. Par leur enseignement et la conduite de cérémonies, ils apportent un soutien aux délinquantes dans leur cheminement.

\section{Rapport Arbour}

Le Rapport Arbour a eu un impact significatif, en matière correctionnelle, pour les délinquantes et pour le SCC dans son ensemble. Mentionnons quelques points parmi les nombreuses recommandations qu'il contenait. En juin 1996, la première sous-commissaire pour les femmes a été nommée. Son mandat touche, entre autres, le développement des politiques et des programmes pour les délinquantes.

Depuis, les équipes d'intervention des établissements régionaux sont composées uniquement de femmes, aucun homme ne pouvant participer ou être témoin d'une fouille à nu.

Suite aux recommandations du Rapport Arbour, le SCC a entrepris une révision complète de sa gestion des unités d'isolement pour l'ensemble des pénitenciers. Dans le cadre de cet exercice, le personnel a reçu une formation concernant les obligations législatives liées à l'isolement préventif. De façon plus générale, les services juridiques ont mis sur pied des séances de formation concernant les aspects juridiques du travail en milieu correctionnel, ces modules faisant maintenant partie des normes nationales de formation.

\section{Dotation mixte}

Depuis l'ouverture des pénitenciers régionaux, toute la question relative à la présence d'hommes comme intervenants de première ligne a fait, et continue de faire, l'objet d'analyses et de débats. Suite aux recommandations du Rapport Arbour, le SCC a confié à une firme indépendante le mandat d'analyser les répercussions de la mixité dans les établissements régionaux. Cette étude, d'une durée de trois ans, s'est terminée avec le dépôt du rapport final de la firme en septembre 2000 (Lajeunesse et al., 2000). 
Les auteures de cette étude ne sont pas favorables à la présence d'hommes comme intervenants de première ligne. Elles se basent principalement sur le raisonnement suivant : la présence d'hommes comme intervenants directs augmente le risque d'atteinte à la vie privée ou d'inconduite sexuelle. Ceci peut aussi raviver des émotions pénibles rattachées à des situations d'abus. Les tenants de cette position ne croient pas à l'effet normalisateur d'un personnel mixte dans un établissement carcéral, alléguant que la nature même de la vie en pénitencier crée un déséquilibre de pouvoir entre détenues et «gardiens». De ce fait, l'autorité que les intervenants de première ligne exercent sur la vie quotidienne des délinquantes se trouve aux antipodes d'une relation basée sur l'égalité.

Tout en reconnaissant le lourd passé d'abus chez plusieurs délinquantes, le SCC continue de soutenir que le pénitencier doit être le reflet de la communauté. Il peut devenir un endroit favorisant l'apprentissage de relations professionnelles positives avec du personnel masculin. Les détenues peuvent aussi y entreprendre une démarche thérapeutique pour travailler sur les blessures du passé, s'il y a lieu, car les programmes et les services psychologiques sont disponibles et accessibles.

Afin de respecter la vie privée des délinquantes, le SCC a mis en place, dès l'ouverture des établissements, un protocole visant à identifier les situations qui doivent être abordées différemment par le personnel masculin. À titre d'exemple, mentionnons que les intervenants masculins ne peuvent effectuer de fouilles par palpation ou de fouilles à nu ou être témoins de ces dernières. Le personnel masculin doit être accompagné d'un membre du personnel féminin pour effectuer une ronde dans les unités d'habitation durant la nuit ou escorter une détenue à l'extérieur du pénitencier, si l'escorte est de type sécuritaire.

Le SCC continue de croire que l'embauche d'un personnel professionnel est la meilleure garantie face aux situations possibles d'atteinte à la vie privée ou aux d'abus.

\section{Programmes}

Parmi les principales difficultés chez les délinquantes, mentionnons la dépendance, la faible estime de soi, l'échec scolaire ou professionnel, le placement et les changements fréquents de famille d'accueil, la vie dans la rue, le commerce du sexe, les tentatives de suicide, l'automutilation et la toxicomanie. 
Afin de répondre aux besoins uniques des délinquantes, le SCC s'est doté d'une stratégie de programmes énonçant les principes qui sous-tendent le développement et la prestation des programmes mis en place dans les établissements régionaux (SCC, 1994). Ces derniers doivent tenir compte de la réalité sociale des femmes, appuyer le développement de l'estime de soi, ainsi que l'autonomie, et être orientés vers la mise en liberté.

De plus, l'élaboration des programmes repose sur des concepts théoriques reconnus. Les approches basées sur les théories concernant l'apprentissage social et cognitif se sont révélées efficaces avec les délinquants, hommes et femmes. Ces théories supportent les principes que les comportements sont appris à travers différentes expériences d'observation, d'imitation, d'exposition répétée et de renforcement. Les programmes correctionnels utilisent les mêmes concepts, dans la mesure où ils permettent aux délinquantes d'observer, de discuter et de pratiquer, à travers différents jeux de rôles, des comportements mieux adaptés aux situations. L'aspect cognitif encourage les délinquantes à entamer un processus de résolution des problèmes, tout en identifiant les émotions liées aux situations difficiles.

Les programmes offerts par chaque établissement peuvent varier légèrement, mais chacun dispense quatre programmes de base.

Les programmes de base du SCC veulent répondre aux facteurs criminogènes (facteurs qui ont joué un rôle dans le comportement criminel) dans le but de réduire les risques de récidive. Un seul type de programmes fait exception à la règle : il s'agit du programme pour survivantes d'abus. Il n'existe, en effet, aucun lien causal direct entre le fait d'avoir été victime de violence, de mauvais traitements ou de traumatisme et le comportement criminel. Cependant, il existe une étroite corrélation entre les expériences d'abus et la délinquance (Blanchette, 2001). Les répercussions de ce type de victimisation atteignent beaucoup d'aspects de la vie d'une personne. Afin d'en souligner l'importance pour les délinquantes, le SCC l'a donc inclu dans ses programmes de base. Ceux-ci se résument de la façon suivante :

1. Programme d'acquisition de compétences psychosociales. Les modules du programme permettent la réflexion sur les valeurs et les attitudes et visent à développer les aptitudes requises pour la résolution des conflits et des problèmes. Ce programme se compose de plusieurs volets, dont la gestion des émotions et de la colère, ainsi que l'apprentissage des aptitudes parentales. 
2. Programmes pour les survivantes d'abus. Ce programme est donné principalement par des organismes de la communauté ayant une compétence dans ce domaine. Il permet aux délinquantes de reconnaître les mécanismes de survie adoptés suite à l'abus, de travailler les émotions liées à ces situations, et de rebâtir l'affirmation et l'estime de soi.

3. Programmes d'alphabétisation et d'éducation. L'obtention du diplôme d'études secondaires est fortement encouragée. La poursuite d'études postsecondaires par correspondance est possible. Le SCC peut en assumer les frais si cet élément est reconnu comme un domaine à travailler au niveau du plan correctionnel de la délinquante.

4. Programmes de lutte contre la toxicomanie. Ce programme possède plusieurs composantes selon la gravité de la dépendance. Il comprend aussi un volet prévention de la rechute, à l'intérieur duquel la délinquante reconnaît les éléments précipitant sa consommation, ainsi que les stratégies visant à mieux gérer les situations à risque. Ce programme est en révision actuellement et la nouvelle version devrait intégrer un volet communautaire.

En s'appuyant sur les renseignements obtenus lors de l'évaluation initiale complète et systématique des facteurs criminogènes et des besoins en matière de programmes, les intervenants établissent un plan correctionnel pour chaque délinquante. Ce plan expose en détail toutes les activités auxquelles la délinquante doit participer au cours de son incarcération, afin de se préparer à une mise en liberté graduelle au moment le plus opportun, en toute sécurité pour le public. Les délinquantes jouent un rôle actif dans la préparation, l'évaluation et la mise en œuvre de leur plan correctionnel.

\section{Programme mère-enfant}

Un des aspects les plus novateurs des établissements régionaux s'avère la possibilité, pour certaines détenues, de vivre avec leur(s) enfant(s) à l'intérieur du pénitencier. D'ailleurs, l'intégration des enfants en milieu carcéral est une pratique établie dans certains pays industrialisés, notamment en Allemagne, où la loi prévoit que les enfants peuvent habiter avec leur mère jusqu'à l'âge scolaire.

La vie en unité d'habitation offre un cadre physique permettant d'y intégrer des enfants. Des chambres sont donc réservées aux enfants à 
l'intérieur de certaines unités. L'objectif du programme mère-enfant est de favoriser et de maintenir les relations positives entre les mères et leurs enfants. De ce fait, la date probable de mise en liberté, ainsi que les projets de sortie de la mère constituent des éléments importants de l'analyse. Toutefois, le meilleur intérêt de l'enfant est toujours le critère prépondérant dans la prise de décision concernant l'acceptation de l'enfant dans le programme de cohabitation.

Deux options principales de cohabitation sont offertes aux participantes au programme mère-enfant : à temps plein ou à temps partiel. L'âge limite pour la cohabitation à temps plein est fixé à quatre ans, et pour la cohabitation à temps partiel, à douze ans. Le SCC a défini des critères d'admissibilité tant pour la détenue que pour l'enfant, de même que des critères d'évaluation. Les programmes d'acquisition de compétences parentales sont obligatoires pour les mères participant au programme. De plus, il faut obtenir la collaboration et le consentement des autorités provinciales concernées, étant donné que les lois en matière de protection de l'enfance sont de compétence provinciale.

La demande d'une détenue voulant participer au programme fait l'objet d'une évaluation rigoureuse. Précisons que la mère doit assumer les responsabilités directes liées à tous les aspects des soins à prodiguer à l'enfant et ce, en tout temps. Elle doit être en mesure de répondre adéquatement aux besoins physiques, sociaux et affectifs de l'enfant. Pour l'assister et aussi lui permettre de suivre certains programmes, elle identifie deux co-détenues qui peuvent agir en tant que gardiennes. Ces dernières font l'objet d'une évaluation complète avant d'être officiellement reconnues comme gardiennes. De plus, comme critère essentiel d'acceptation, la détenue doit avoir une ressource dans la communauté pouvant prendre charge de l'enfant au besoin, et ainsi permettre à l'enfant de quitter le pénitencier de façon régulière pour des activités extérieures.

Le pavillon de ressourcement a accueilli en moyenne quatre enfants par mois durant les dix derniers mois. Le taux de participation à l'intérieur des établissements régionaux est plus irrégulier. Sur une base nationale, environ deux enfants par mois sont hébergés à temps plein dans l'ensemble des établissements régionaux, et autant le sont à temps partiel.

Mais ces chiffres ne reflètent pas le niveau d'activités de ce secteur. Pour plusieurs détenues, la préservation du lien avec leurs enfants est nécessaire à leur équilibre psychologique et émotif. C'est pourquoi la cohabitation n'est qu'un aspect du programme. D'autres options visant à maintenir les contacts sont disponibles selon les besoins de la mère et 
la situation de l'enfant, telles que les visites prolongées d'une journée, le programme de visite familiale privée et des rencontres sous supervision, si requises par le personnel des services sociaux.

\section{Stratégie d'intervention intensive}

En 1995, les établissements d'Edmonton et de Nova ont été les premiers à accueillir des détenues. Une série d'incidents à l'établissement d'Edmonton, dont des évasions, des gestes automutilatoires et un suicide qui, après enquête, s'est avéré être un meurtre, ont amené le SCC à remettre en question certaines orientations prises suite à la publication du rapport La création de choix. Il est apparu évident que l'approche axée sur la vie communautaire et la responsabilisation ne répondait pas aux besoins et aux risques que représentait une petite partie de la population carcérale. L'hypothèse soutenue par les auteurs du rapport voulant qu'un séjour de courte durée à l'unité d'encadrement, renforcé (aile sécuritaire des établissements régionaux) par un plan d'intervention, aurait permis de réintégrer rapidement dans la population régulière les délinquantes présentant des problématiques plus sévères, s'est révélée fausse. En effet, l'expérience a montré que certaines délinquantes ont besoin d'un plan d'intervention à plus long terme et plus structuré.

Donc, à la suite de ces incidents, les délinquantes ayant une cote de sécurité maximum ont été transférées dans une section d'un établissement pour hommes de leur région respective, sauf en Ontario où la prison des femmes est demeurée ouverte pour les héberger. Précisons que toutes ces unités sont complètement autonomes et séparées de la population masculine. À l'automne 2001, environ $9 \%$ de la population féminine purgeant une sentence fédérale avait une cote de sécurité maximum, soit 36 détenues.

Dès le départ, le SCC a convenu que la localisation d'unités de femmes dans des établissements pour hommes devait être une situation temporaire. Une stratégie de gestion et de logement à long terme pour les délinquantes dites à sécurité maximum et celles présentant des problèmes de santé mentale se devait d'être élaborée. Le SCC s'est donc adjoint des experts du milieu afin d'alimenter ses réflexions et de valider ses hypothèses de travail. L'orientation proposée devait tenir compte du niveau d'encadrement nécessaire, des programmes adaptés aux besoins des délinquantes, ainsi que de la formation requise pour le personnel.

Approuvée par le Conseil du Trésor en mai 1999, la stratégie d'intervention intensive comporte deux approches distinctes, soit celle pour 
le développement d'un milieu de vie structuré et celle pour la mise en place d'une unité de garde en milieu fermé.

\section{a) Milieu de vie structuré}

Le milieu de vie structuré (MVS) offre un type de traitement distinct pour les délinquantes ayant une cote de sécurité minimum ou moyenne, mais présentant de graves carences intellectuelles ou des troubles de santé mentale.

Le SCC a demandé à des professionnels qui ne relèvent pas du gouvernement d'étudier les besoins précis des délinquantes ayant des problèmes de santé mentale. Leurs rapports ainsi que leurs recommandations ont guidé le SCC dans l'élaboration de cette stratégie. C. G. Whitehall (1995) et M. Rivera (1996) ont toutes deux évalué les besoins particuliers de cette clientèle et sont parvenues aux mêmes conclusions : certaines délinquantes ont besoin d'intervention intensive et tireraient avantage d'un environnement structuré et organisé selon le principe de foyer de groupe.

Par la suite, A. D. Warner (1998) a été mandaté afin de proposer une approche destinée aux délinquantes ayant des besoins spéciaux. Son rapport a joué un rôle déterminant dans l'élaboration des programmes pour le milieu de vie structuré. Le $\mathrm{D}^{\mathrm{r}}$ Warner a souligné, notamment, que les délinquantes dites à besoins spéciaux ne constituent pas un groupe homogène. En effet, chez cette clientèle, plusieurs types de problèmes sont présents, notamment la toxicomanie, le comportement suicidaire ou l'automutilation, le comportement agressif, les difficultés en matière de relations humaines et les compétences psychosociales insuffisantes. De plus, ces femmes ont des besoins très différents pouvant même être incompatibles, créant ainsi des situations explosives. Les délinquantes souffrant de carences intellectuelles, par exemple, ont de la difficulté à tolérer le voisinage de délinquantes qui manifestent une grande détresse émotive et sont sujettes à des accès de colère ou adoptent un comportement destructeur.

Il a démontré l'importance pour le SCC de faire appel à une équipe interdisciplinaire possédant de l'expérience en santé mentale, pour s'assurer de l'efficacité des programmes et de la cohérence des interventions. Le SCC a entériné cette orientation.

La mise en œuvre des recommandations contenues dans le rapport $\mathrm{du} \mathrm{D}^{\mathrm{r}}$ Warner a nécessité le recrutement et la formation d'une équipe de travail, ainsi que la construction, dans chacun des établissements régionaux, d'une unité d'habitation comprenant des zones d'habitation et des 
locaux pour le personnel et pour les programmes. Cette unité de huit chambres se divise en deux parties distinctes, ce qui permet plus de flexibilité pour le traitement des clientèles incompatibles.

Deux approches spécialisées ont été mises en place pour répondre aux besoins variés de cette population, soit la thérapie dialectique du comportement et la thérapie de réadaptation psychosociale.

La thérapie dialectique du comportement est une approche basée sur des recherches empiriques. Elle est largement reconnue pour ses résultats positifs auprès d'une clientèle ayant des agissements propres au désordre de personnalité-limite (par exemple des comportements autodestructeurs, des problèmes relationnels et des distorsions cognitives). Ces délinquantes ont tendance à réagir immédiatement et souvent de façon extrême aux événements. Ces réponses interferent avec leur processus cognitif et alimentent une chaîne de réactions qui les entraînent encore plus loin dans une série de comportements inappropriés. Cette thérapie offre une approche prometteuse avec les femmes aux prises avec de telles difficultés. Quant à la réadaptation psychosociale, c'est une méthode qui convient aux personnes qui manquent de compétences de base ou qui ont un déficit cognitif. Les interventions sont basées sur une approche sociale d'apprentissage à l'intérieur d'un environnement structuré. Elles incorporent différentes techniques, telles que l'apprentissage d'aptitudes et le renforcement positif.

La construction, la sélection du personnel, sa formation et la mise en œuvre des programmes reliés au milieu de vie structuré ont été complétées durant l'automne 2001.

\section{b) Unité de garde en milieu fermé}

En fonction de recherches antérieures et de l'expérience acquise, le SCC a élaboré un modèle d'intervention intensive afin de répondre aux besoins des délinquantes qui ont une cote de sécurité maximale et utilisent davantage la violence en milieu carcéral (McDonagh, 1999; Morin 1999). La priorité est de les motiver, afin qu'elles apprennent à maîtriser leurs comportements agressifs ou impulsifs. Tout comme le groupe de délinquantes ayant des besoins spéciaux, ce groupe n'est pas homogène. Certaines peuvent avoir un comportement antisocial, tandis que d'autres ont de graves troubles affectifs ou psychologiques.

La stratégie d'intervention nécessaire à l'encadrement de ces délinquantes se compose de plusieurs éléments, dont un niveau de sécurité 
statique plus élevé, un personnel bien formé, une approche correctionnelle interdisciplinaire et une sécurité dynamique très présente. L'équipe interdisciplinaire devra établir des plans individuels d'intervention axés sur la gestion du comportement. Les membres du personnel des unités de garde en milieu fermé recevront la même formation en santé mentale que leurs collègues de l'équipe de travail du milieu de vie structuré. L'accent sera mis sur une stricte gestion du comportement et sur l'acquisition de compétences par les délinquantes. À cet effet, la thérapie dialectique du comportement, ainsi que la réadaptation psychosociale, seront des approches utilisées. Le personnel joue un rôle important dans la création et le maintien d'un environnement qui renforcera les comportements positifs et les efforts de changement.

Deux programmes sont actuellement mis à l'essai dans les unités de délinquantes ayant une cote de sécurité maximum. L'un d'eux, Esprit de guerrier, a été conçu spécialement pour les délinquantes autochtones. Ce programme les amène à réfléchir aux actes de violence qu'elles ont commis et à comprendre comment la violence a influencé leur vie. Il se divise en plusieurs composantes, dont la sensibilisation culturelle, la prise de conscience de sa colère et de sa violence, l'acquisition de compétences et l'apprentissage cognitif. L'autre programme de traitement intensif intègre des éléments de la thérapie dialectique du comportement et favorise chez les participantes une meilleure compréhension de leurs comportements violents, dysfonctionnels et autodestructeurs. Les modules du programme incluent l'aspect autobiographique, les infractions criminelles, l'empathie envers la victime, ainsi que l'acquisition d'aptitudes émotionnelles et interpersonnelles visant à prévenir les comportements violents. Ces programmes seront disponibles dans les établissements régionaux.

L'unité à encadrement renforcé des établissements régionaux sera rénovée en fonction du modèle d'intervention intensive. La capacité cellulaire de ces unités varie selon les régions (entre 10 et 15 cellules). De plus, des locaux sont prévus pour les programmes à l'intérieur de l'unité, bien que les détenues puissent avoir accès au bâtiment principal pour d'autres activités, mais seulement si le risque est acceptable et sous la supervision du personnel.

La rénovation, ainsi que l'embauche et la formation du personnel, seront terminées en 2002, ce qui permettra de mettre fin à la cohabitation dans les établissements pour hommes, à l'exception de l'unité des femmes du Centre psychiatrique régional de la région des Prairies, qui 
restera ouverte en raison de sa particularité. Accrédité comme un centre de traitement, le Centre psychiatrique régional offre un programme spécialisé pour les cas aigus de santé mentale. Il aura une vocation nationale.

\section{Délinquantes sous surveillance dans la communauté}

Le tableau 2 illustre l'évolution de la population sous surveillance depuis novembre 1995.

Depuis 1995, le nombre de délinquantes en communauté a augmenté chaque année sur le plan national, à l'exception de la dernière année où une légère diminution a été enregistrée. Tout comme au niveau de la population incarcérée, les régions de l'Atlantique et des Prairies ont connu les plus fortes augmentations. Il est aussi important de souligner que la proportion de délinquantes en communauté, comparée à la proportion de délinquantes incarcérées, est passée d'environ 51,9\% en 1996 à environ $56,9 \%$ en 2001 .

En 2001, environ $16 \%$ des délinquantes en supervision bénéficiaient d'une semi-liberté, $72 \%$ d'une libération conditionnelle totale et $12 \%$ d'une libération d'office.

Comparées aux hommes, les délinquantes en libération conditionnelle se distinguent de la façon suivante (données de janvier 2001) :

- Comparativement aux délinquants, elles bénéficient davantage d'une libération conditionnelle totale (environ $72 \%$ par rapport à $51 \%$ pour les hommes). De plus, $61 \%$ des femmes sont dans la catégorie «risque faible», comparativement à $44 \%$ pour les hommes.

TA B LEA U 2

Tendances - Population en communauté

\begin{tabular}{|c|c|c|c|c|c|c|}
\hline & Atlantique & Québec & Ontario & Prairies & Pacifique & Total \\
\hline Nov. 1995 & 19 & 86 & 136 & 56 & 34 & 331 \\
\hline Nov. 1996 & 25 & 85 & 141 & 70 & 35 & 356 \\
\hline Nov. 1997 & 30 & 92 & 175 & 75 & 39 & 411 \\
\hline Nov. 1998 & 40 & 101 & 191 & 89 & 46 & 467 \\
\hline Nov. 1999 & 44 & 101 & 208 & 98 & 47 & 498 \\
\hline Nov. 2000 & 40 & 89 & 191 & 127 & 55 & 502 \\
\hline Nov. 2001 & 34 & 96 & 186 & 126 & 47 & 489 \\
\hline
\end{tabular}

Systèmes intégrés de rapports du SCC, novembre 2001 
- Leur motivation ainsi que leur potentiel de réinsertion sociale sont plus élevés que chez les hommes. En effet, $77 \%$ des délinquantes, comparativement à $61 \%$ des délinquants, sont dans la catégorie «motivation élevée» et $75 \%$ d'entre elles sont dans la catégorie «potentiel élevé de réinsertion sociale», comparativement à $55 \%$ pour les hommes.

- Comparativement aux hommes, elles présentent des besoins plus élevés dans les domaines des relations maritales/familiales et de l'emploi. En contrepartie, elles ont des besoins moins élevés pour le domaine lié à l'attitude, car elles adoptent plus facilement des valeurs pro-sociales.

- La clientèle féminine est ouverte à l'intervention. La délinquante demande de l'aide et du soutien lorsqu'elle éprouve des difficultés.

- La population féminine en libération conditionnelle est dispersée. Environ le tiers des délinquantes vivent en dehors des grands centres.

En ce qui a trait aux délinquantes en communauté, les principes suivants guident le SCC :

- La réinsertion sociale est un processus continu qui débute à la première journée d'incarcération. Il faut s'assurer que la mise en liberté se fera au moment opportun. Afin de répondre aux besoins spécifiques des délinquantes, un agent de la communauté participe en début de sentence à l'examen du soutien nécessaire à une réinsertion sociale réussie.

- La gestion de la sentence comprend l'examen des programmes et services qui seront dispensés en établissement et ceux qui le seront en communauté. La participation de partenaires, tels que les intervenants des maisons de transition, les responsables de la protection de la jeunesse, les bénévoles, les aînés et toute autre personne significative, est essentielle au développement d'un plan de mise en liberté réaliste. La détenue elle-même fait partie intégrante de cette équipe.

- Le but à long terme de la surveillance est de favoriser l'appartenance des délinquantes aux groupes de femmes de leur milieu. Cette intégration aux ressources existantes de leur communauté leur permettra d'établir des liens positifs qui se maintiendront bien après l'expiration de leur mandat.

- Tout en prenant en considération la sécurité du public, des solutions de rechange à l'incarcération sont envisagées lors de manquements 
aux conditions spéciales. Dans ces situations, la réincarcération est utilisée comme mesure de dernier recours après que d'autres solutions permettant de gérer le risque ont été évaluées.

De façon plus concrète, le SCC s'est doté, au cours des dernières années, d'un réseau de logement mieux adapté aux besoins des délinquantes. Les places d'hébergement en communauté sont en nombre suffisant. De plus, d'autres avenues ont été explorées et mises sur pied, telles qu'un réseau de foyers privés ou d'appartements satellites. Ces formes innovatrices d'hébergement permettent de répondre aux besoins particuliers de certaines délinquantes ou de répondre aux besoins en région rurale.

L'abus d'alcool et de drogues constitue un des éléments expliquant la suspension ainsi que la révocation de la libération conditionnelle dans de nombreux cas. Une attention particulière est donc portée aux programmes touchant ces dimensions. Le SCC possède des contrats de service avec des centres de traitement intensif. De plus, le SCC poursuit le développement d'un programme national de prévention de la rechute.

Le SCC favorise l'établissement de partenariats avec les autres paliers de gouvernement, le secteur communautaire en justice pénale, ainsi qu'avec le réseau des ressources pour les femmes, afin de permettre la réflexion en ce qui touche la criminalité féminine, l'échange d'information et le développement de ressources ou de programmes. Des initiatives, telles que des tables de concertation, des groupes de travail multisectoriels et des comités interjuridictions, sont encouragées.

Malgré le fait que la grande majorité des délinquantes terminent avec succès leur période de surveillance, certaines d'entre elles voient leur libération conditionnelle suspendue ou révoquée.

Les données du tableau 3 proviennent d'un rapport spécial sur les délinquantes, s'étalant sur la période de 1995-1996 à 1999-2000, produit par la division de la Mesure du rendement de la Commission nationale des libérations conditionnelles (2000). Les chiffres du tableau qui suit sont basés sur les périodes de supervision complétées et non sur le nombre de délinquantes.

Ce rapport contient aussi une comparaison entre les hommes et les femmes sous supervision. Les éléments suivants sont relevés pour les années comprises entre 1995 et 2000 :

- Les délinquantes ont des taux d'achèvement plus élevés que les délinquants pour toutes les formes de mise en liberté (semi-liberté : 85,9\% pour les délinquantes par rapport à $82,2 \%$ pour les hommes; libé- 
TAB LEA U 3

Résultats des mises en liberté des délinquantes de 1995-1996 à 1999-2000

\begin{tabular}{|c|c|c|c|c|c|c|}
\hline Résultats & \multicolumn{2}{|c|}{ Semi-liberté } & \multicolumn{2}{|c|}{$\begin{array}{l}\text { Libération } \\
\text { conditionnelle totale }\end{array}$} & \multicolumn{2}{|c|}{$\begin{array}{l}\text { Libération } \\
\text { d'office }\end{array}$} \\
\hline \multirow[b]{2}{*}{$\begin{array}{l}\text { Achèvement } \\
\text { Révocation pour violation } \\
\text { des conditions }\end{array}$} & Nbre & $\%$ & Nbre & $\%$ & Nbre & $\%$ \\
\hline & 707 & 85,9 & 436 & 77,0 & 303 & 63,7 \\
\hline \multicolumn{7}{|c|}{ Récidive (révocation pour infraction) } \\
\hline Sans violence & 31 & 3,8 & 37 & 6,5 & 38 & 8,0 \\
\hline Avec violence & 10 & 1,2 & 4 & 0,7 & 11 & 2,3 \\
\hline Total des récidives & 41 & 5,0 & 41 & 7,2 & 49 & 10,3 \\
\hline $\begin{array}{l}\text { Total des semi-libertés } \\
\text { terminées }\end{array}$ & 823 & $100 \%$ & 566 & $100 \%$ & 476 & $100 \%$ \\
\hline
\end{tabular}

ration conditionnelle totale : $77 \%$ pour les délinquantes comparativement à $68,7 \%$ pour les délinquants; libération d'office : $63,7 \%$ pour les délinquantes par rapport à $58,3 \%$ pour les délinquants).

- Les délinquantes ont moins de révocations de la libération pour une nouvelle infraction que les hommes sous surveillance. Les différences sont plus importantes dans la catégorie de la libération conditionnelle totale (révocation pour une infraction sans violence : 6,5\% par rapport à $12,3 \%$ pour les hommes; révocation pour une infraction avec violence : $0,7 \%$ comparativement à $2,3 \%$ pour les hommes).

Dans l'ensemble, la performance des délinquantes en libération conditionnelle est meilleure que celle des délinquants. Toutefois, la zone potentielle d'amélioration au chapitre de la surveillance se situe au niveau des révocations sans nouvelle infraction. L'expérience indique que souvent la délinquante a fait l'objet de mesures de rechange à l'incarcération avant de voir sa libération conditionnelle révoquée. Souvent, des difficultés liées à la consommation d'alcool ou de drogues justifient la révocation.

\section{Défis}

Depuis les dix dernières années, plusieurs initiatives concernant les délinquantes ont été mises en place. Cependant, les prochaines années seront 
tout aussi importantes dans la poursuite du développement du modèle correctionnel pour les délinquantes.

- L'intégration des détenues ayant une cote de sécurité maximum à l'intérieur des établissements régionaux demeure un défi à relever. La crainte exprimée par certains partenaires est que l'arrivée de ces délinquantes va créer une pression qui aura comme conséquence d'augmenter les mesures sécuritaires pour l'ensemble de la population. Le SCC a pour objectif d'appliquer un régime différent pour les détenues à sécurité maximum afin d'éviter une telle situation.

- Le SCC devra évaluer la mise en œuvre des programmes spécialisés pour les délinquantes ayant des problèmes de santé mentale qui bénéficient du milieu de vie structuré et, de façon plus précise, mesurer les résultats obtenus en matière de réintégration de cette clientèle dans la population régulière de l'établissement et, subséquemment, dans la communauté.

- La sélection du personnel et, plus particulièrement, celle des intervenants de première ligne constituent un des éléments essentiels au maintien du modèle correctionnel souhaité pour les établissements régionaux. Malgré les contraintes opérationnelles, le SCC doit poursuivre cette orientation afin de s'assurer que les intervenants ont les aptitudes requises pour intervenir auprès de la clientèle féminine et se sentent à l'aise avec le modèle correctionnel proposé.

- Le gouvernement fédéral a pris un engagement important en voulant ramener, d'ici une génération, le taux d'incarcération de la population autochtone au même niveau que celui de la population non autochtone. Beaucoup reste à faire en ce qui concerne le développement de programmes adaptés à la réalité autochtone. La recherche demeure embryonnaire en ce qui a trait aux connaissances entourant le syndrome d'alcoolisme fotal et surtout les mesures de traitement de ce problème. La population de délinquants et de délinquantes autochtones semble touchée davantage par ce genre de situation.

- Certaines délinquantes éprouvent des difficultés à maintenir les acquis réalisés en établissement en ce qui concerne leur dépendance face à l'alcool ou à la drogue. Un nouveau programme de toxicomanie est en voie d'élaboration; il va intégrer un volet communautaire. Un suivi à cet égard doit se faire afin de mesurer les conséquences de cette initiative sur le taux de suspension et de révocation. 


\section{Conclusion}

Le SCC est une des composantes du système de justice pénale. Son mandat, clairement défini dans sa Mission, indique que «[1]e SCC [...] contribue à la protection de la société en incitant activement et en aidant les délinquants à devenir des citoyens respectueux des lois, tout en exerçant sur eux un contrôle raisonnable, sûr, sécuritaire et humain» (p. 4). Le personnel du SCC travaille en fonction de la sentence imposée par le juge en croyant au potentiel de changement des délinquants et des délinquantes et en étant convaincu que la presque totalité d'entre eux retourneront éventuellement en communauté.

Selon certains de nos partenaires, l'effet «pervers» du Rapport $L a$ création de choix a été la multiplication des prisons, mais le SCC, compte tenu de son mandat législatif, n'a jamais remis en question l'existence des pénitenciers et n'a donc jamais poursuivi cet objectif. La régionalisation des établissements est, de fait, l'une des pierres angulaires du nouveau modèle proposé dans le rapport, en ce qu'elle rapproche les délinquantes de leur soutien familial et de leur communauté d'origine. Au cours de la dernière décennie, le SCC a maintenu le cap vers de meilleurs programmes et services adaptés aux délinquantes. Des programmes répondant aux besoins uniques des délinquantes ont été élaborés et mis en place. De plus, une attention particulière a été portée à celles qui ont des besoins spéciaux. Ce travail a été accompli grâce au professionnalisme du personnel en place et à son engagement à l'égard du modèle opérationnel préconisé par le SCC.

\section{Références}

Arbour, L. (1996). Commission d'enquête sur certains événements survenus à la Prison des femmes de Kingston. Ottawa : Ministre des Approvisionnements et Services Canada.

Blanchette, K. (2001). Classifying female offenders for effective intervention: Application of the case-based principles of risk and need. Unpublished comprehensive paper submitted for Ph.D (Psychology), Carleton University, Ottawa, Ontario.

Commission nationale des libérations conditionnelles. Rapport de surveillance $d u$ rendement 1999-2000.

Lajeunesse, T., Jefferson, C., Nuffield, J., \& Majury, D. (2000). Projet de vérification de la dotation mixte : troisième et dernier rapport annuel. Ottawa : Service correctionnel du Canada.

Gouvernement du Canada (1992). Loi sur le système correctionnel et la mise en liberté sous condition (ch. 20). 
McDonagh, D. (1999). Détenues sous responsabilité fédérale - Projet d'entrevue de détenues dites à sécurité maximale : faire du temps sans se laisser faire par le temps. Ottawa : Service correctionnel du Canada.

Morin, S. (1999). Les détenues autochtones à sécurité maximale purgeant une peine fédérale - Que sont devenues les promesses de la création de choix? Ottawa : Service correctionnel du Canada.

Rivera, M. (1996). Donnez-nous une chance — L'évaluation des besoins : les ressources en santé mentale pour les femmes sous responsabilité fédérale dans les établissements régionaux. Ottawa : Service correctionnel du Canada.

Service correctionnel du Canada. (1990). La création de choix : rapport du groupe d'étude sur la femme purgeant une peine fédérale. Ottawa : Ministère des approvisionnements et Services.

Service correctionnel du Canada. (1994). Stratégie des programmes correctionnels à l'intention des femmes purgeant une peine fédérale. Ottawa : Service correctionnel du Canada

Service correctionnel du Canada. (1997). La mission du Service correctionnel $d u$ Canada. Ottawa : Ministère des Approvisionnements et Services Canada.

Whitehall, C.G. (1995). Profil de santé mentale des détenues sous responsabilité fédérale de la région de l'Atlantique et stratégie d'intervention. Ottawa : Service correctionnel du Canada.

Warner, A. (1998). Mise en auvre de choix aux établissements régionaux : propositions de programmes pour les délinquantes ayant des besoins spéciaux. Ottawa : Service correctionnel du Canada. 